\title{
Interleukin- I 0 polymorphisms in Spanish IgA deficiency patients: a case-control and family study
} Javier Ortiz ${ }^{1}$, Miguel Fernández-Arquero1 ${ }^{1}$, Elena Urcelay ${ }^{1}$, Raquel LópezMejías ${ }^{1}$, Antonio Ferreira ${ }^{2}$, Gumersindo Fontán ${ }^{2}$, Emilio G de la Concha ${ }^{1}$ and Alfonso Martínez*1

\author{
Address: ${ }^{1}$ Clinical Immunology Department, Hospital Clínico San Carlos, Madrid and ${ }^{2}$ Immunology Section, Hospital Universitario La Paz, \\ Madrid \\ Email: Javier Ortiz - ortizesa@yahoo.es; Miguel Fernández-Arquero - mfernandeza.hcsc@salud.madrid.org; \\ Elena Urcelay - eurcelay.hcsc@salud.madrid.org; Raquel López-Mejías - ralome@wanadoo.es; \\ Antonio Ferreira - aferreira.hulp@salud.madrid.org; Gumersindo Fontán - gfontan.hulp@salud.madrid.org; Emilio G de la \\ Concha - egomezdela.hcsc@salud.madrid.org; Alfonso Martínez* - alfmdoncel@terra.es \\ * Corresponding author
}

Published: 27 June 2006

BMC Medical Genetics 2006, 7:56 doi: 10.1 | 86/147|-2350-7-56

This article is available from: http://www.biomedcentral.com/I47|-2350/7/56

(C) 2006 Ortiz et al; licensee BioMed Central Ltd.

This is an Open Access article distributed under the terms of the Creative Commons Attribution License (http://creativecommons.org/licenses/by/2.0), which permits unrestricted use, distribution, and reproduction in any medium, provided the original work is properly cited.
Received: 27 January 2006

Accepted: 27 June 2006

\begin{abstract}
Background: IgA deficiency (IgAD) is the most common primary immunodeficiency in Caucasians. Genetic and environmental factors are suspected to be involved in the development of the disease. Interleukin-10 (IL-I0) is a cytokine with stimulatory activity on immunoglobulin production and it may be an important regulator in IgAD pathogenesis. The IL-I0 gene contains several single nucleotide polymorphisms (SNPs) and two polymorphic microsatellites located in the 5'-flanking region. Our aim was to ascertain if any of these polymorphic markers are associated or linked to IgAD in Spanish patients.
\end{abstract}

Methods: We genotyped 278 patients with IgAD and 573 ethnically matched controls for the microsatellites IL-IOR and IL-IOG and for three single nucleotide polymorphisms at positions $1082,-819$ and -592 in the proximal promoter of the gene. We also included in this study the parents of 194 patients in order to study the IL-10 haplotypes transmitted and not transmitted to the affected offspring.

Results: The only allele where a significant difference was observed in the comparison between IgA deficiency patients and controls was the IL-IOGI2 allele $(O R=1.58$ and $p=0.021)$. However, this $p$ value could not withstand a Bonferroni correction. None of the IL-IOR or promoter SNP alleles was found at a different frequency when patients were compared with controls.

Conclusion: Our data do not show any significant difference in IL-10 polymorphism frequencies between control and IgAD patient samples. Their haplotype distribution among patients and controls was also equivalent and therefore these microsatellites and SNPs do not seem to influence IgAD susceptibility. 


\section{Background}

IgA deficiency (IgAD) is the most common primary immunodeficiency in Caucasians and is sometimes associated with deficiency of IgG2, IgG3, IgG4 and IgE [1]. Genetic studies indicate that IgAD may have an inherited component and the disease is associated with certain susceptibility genes located in the major histocompatibility complex region [2,3]. Celiac disease and selective IgA deficiency are frequently associated, and share a similar genetic background [4,5]. Environmental factors also play a role in the occurrence of IgAD. The most usual consequence of this disease is an increased susceptibility to infection [6].

Although the basic immunologic defect that underlies IgAD is unknown, a number of in vitro immunological alterations have been identified: arrest in the $\mathrm{B}$ cell differentiation pathway [7], and a decrease in T helper lymphocyte function $[8,9]$.

IL-10 is a regulatory cytokine that has several functions; it is a potent stimulator of NK cells [10] and has stimulatory effects in B-cell proliferation but due to what has been termed "the dual role" of IL-10, it has also inhibitory effects and suppresses the proinflammatory functions of antigen-presenting cells (APCs) by antagonizing expression of costimulatory molecules, the release of proinflammatory cytokines and, in general, APC maturation [1114]. In addition to inhibiting APC maturation, IL-10 prolongs their capability for antigen uptake while simultaneously postponing their migration to draining lymph nodes. Finally, the IL-10 knockout mouse develops chronic enterocolitis [15], highlighting its important role as modulator of mucosal immunity.

Peripheral blood mononuclear cells (PBMC) from IgAD patients cultured in the CD40/SAC (Staphylococcus aureus Cowan) system produced IgM and IgG, but not IgA. The addition of IL-10 to the cultures enhanced the production of IgM and IgG and most strikingly induced the production of high amounts of IgA. Thus, IL-10 can remove the block in B cell differentiation and allows B cells from IgAD patients to differentiate into IgA secreting cells [16].

The IL-10 gene is located on the long arm of human chromosome 1 and its proximal promoter contains three single nucleotide polymorphisms (SNPs) at positions -1082 (A/G), -819 (T/C) and -592 (C/A) (17-20). Only three haplotypes have been described in Caucasian populations: ATA, ACC and GCC. The production level of this cytokine is mainly determined by the mRNA synthesis rate, which in turn depends on these promoter polymorphisms [17]. The 5'-flanking region also contains two microsatellites: IL-10G and IL-10R [18,19]. The haplotypes formed by both microsatellites also correlate with cytokine production, presumably due to the linkage disequilibrium between promoter and microsatellite alleles forming extended haplotypes [20,21].

There is only a previous study analyzing polymorphisms of IL-10 polymorphisms in IgA deficiency [22], but the small sample size did not allow reaching definite conclusions. The present study is aimed at performing a comprehensive association analysis of IL-10R and IL-10G microsatellites and IL-10 proximal promoter SNPs in a large Spanish sample of IgA deficiency patients. We found no evidence of association of IL-10 polymorphisms to IgAD in the Spanish population in our study.

\section{Methods}

The study group consisted of 278 Caucasian Spanish patients (45\% female) with IgA deficiency. They were diagnosed by serology criteria (serum IgA level below 0.05 $\mathrm{g} / \mathrm{l}$ ). The samples were recruited from a single centre (La Paz Hospital, Madrid, Spain) and most of them have been included in previous studies from our group. Informed consent was obtained from all the participants. This study was approved by the Ethics Committee of the Hospital Clínico San Carlos, and it is in compliance with the Helsinki declaration. The patients can be distributed in different groups, according to their clinical symptomatology: allergy $(16 \%)$, autoimmune disease $(17 \%)$, celiac disease $(6 \%)$, infectious diseases $(13 \%)$, psoriasis $(4 \%)$, other (44\%) or according to the presence of auto-antibodies: anti IgA antibodies (16\%), antinuclear antibodies (11\%), anticardiolipins (3\%), microsomal antibodies (3\%) and no antibodies $(67 \%)$. We could also obtain progenitor samples of 194 patients (usually trios father-motherpatient). A group of 573 healthy white, unrelated subjects (50.8\% women) from the Madrid region (mainly Hospital employees and blood donors) was selected as controls. Many of the patient and familiy samples have been included in previous studies of our group in the field of IgAD genetics [23-25]

IL-10G and IL-10R microsatellites were amplified using primers and conditions previously described [20]. Samples were subsequently denatured and run on an $\mathrm{ABI}$ Prism 3100 automatic sequencer (Applied Biosystems, Foster City, Calif.). Each sample included an internal size standard (HD400 ROX; Applied Biosystems) to achieve a highly consistent measure. The results were analyzed using GeneMapper v3.0 (Applied Biosystems).

There are only three IL-10 promoter haplotypes (GGC, ATA, ACC) described $[21,26,27]$ in our population. That is why we have only analyzed the first $(-1082)$ and the third (-592) position in the IgAD patients and we then obtained the second position (-819) by a simple deduction. The SNP study has been performed using TaqMan 
Table I: Phenotypic frequencies of IL-IOR and IL-I0G microsatellites in patients with IgA deficiency $(n=278)$ and controls $(n=573)$

\begin{tabular}{lcccc}
\hline IL-IOR & Patients & $\%$ & Controls & $\%$ \\
\hline 2 & 266 & 96 & 552 & 96 \\
3 & 97 & 35 & 198 & 34 \\
4 & 11 & 4 & 19 & 3.3 \\
IL-I0G & Patients & $\%$ & Controls & $\%$ \\
7 & 13 & 5 & 16 & 3 \\
8 & 12 & 4 & 34 & 6 \\
9 & 150 & 54 & 305 & 53 \\
10 & 37 & 13 & 75 & 13 \\
11 & 66 & 24 & 140 & 24 \\
12 & 47 & 17 & 63 & 11 \\
13 & 109 & 39 & 262 & 46 \\
14 & 44 & 16 & 81 & 14 \\
15 & 7 & 3 & 18 & 3 \\
\hline
\end{tabular}

probes (C__1747360_10 for -1082 and C__1747363_10 for -592) under conditions recommended by the manufacturer. The samples were read by two observers and some samples with known results were included in the analysis as quality controls. Healthy controls were typed (all three positions) as described elsewhere [26]. Concordance between the two techniques was total.

The frequencies of each marker allele in patients and controls were compared by a standard Chi-Square test. The comparisons between haplotypes transmitted and not transmitted were also done by this method. For each locus, a Bonferroni correction was applied. Only alleles found at a frequency higher than 3\% were taken into account for correction purposes. A result was considered significant if $\mathrm{P}_{\mathrm{c}}<0.05$. The present study has a statistical power of $80 \%$ to detect an association with a relative risk of 1.5 for an allele with a phenotypic frequency of 0.2 .

\section{Results}

Table 1 shows the results from the IL-10R and IL-10G microsatellite analysis. The alleles found at a higher frequency were IL-10R2 for IL-10R, and IL-10G9 and IL-10G13 for IL-10G, as described in other Caucasian populations. The IL-10R1, IL-10G1-G6 and IL-10G16-G17 alleles were extremely rare, also in keeping with previous reports.

Table 2: Phenotypic frequencies of IL- 10 promoter haplotypes in patients with IgA deficiency $(n=27 I)$ and controls $(n=529)$

\begin{tabular}{ccccc}
\hline Allele & Patients & $\%$ & Controls & $\%$ \\
\hline ATA & 121 & 45 & 235 & 44 \\
ACC & 150 & 55 & 287 & 54 \\
GCC & 179 & 66 & 332 & 63 \\
\hline
\end{tabular}

In the comparison between IgA deficiency patients and controls the only significantly different value observed was the frequency of IL-10G12 allele, higher in patients, $(\mathrm{OR}=1.58, \mathrm{p}=0.021)$. However, this $\mathrm{p}$ value could not withstand a Bonferroni correction. None of the IL-10R alleles was found at a different frequency in the group of patients when compared with controls.

Table 2 displays the results observed in IL-10 promoter polymorphisms. The IL-10 promoter haplotype with higher frequency, both in patients and in controls, was $\mathrm{G}(\mathrm{C}) \mathrm{C}$. We did not observe any significant difference between patients and controls when promoter frequencies were compared.

Table 3 shows the extended haplotypes comprising IL10R, IL10G microsatellites and IL-10 gene promoter SNPs more frequently found in families, sorted in transmitted to the patients and not transmitted to them. The extended haplotype more frequent was IL10R2-IL10G9-A(T)A. Haplotypes extremely rare in our study are included under the "Others" heading. As it can be observed, there are more transmitted haplotypes in total (388) than nontransmitted (332). This is because in absence of one progenitor we could not obtain the haplotype non-transmitted by that progenitor, unless an additional family member was available. We can observe a greater proportion of IL10R2-IL10G13-G(C)C haplotype transmitted than no transmitted $(\mathrm{OR}=2.0, \mathrm{p}=0.024)$, but again the $\mathrm{p}$ value failed to reach a statistically significant level after Bonferroni correction.

Due to the well-known association of IgAD with HLA haplotypes (marked by DR1 or DRB1*0102, DR3 and DR7 as risk factors, and DR2-DQ6 as a protection haplotype), we performed HLA-stratified analyses of the IL-10 polymorphisms, but no interaction of IL-10 alleles with any predisposition-related HLA allele or haplotype was found (data not shown).

\section{Discussion}

In this study we have undertaken an association analysis of IL-10 polymorphisms (IL-10R and IL-10G microsatellites and promoter SNPs) in a large sample of Spanish patients with IgA deficiency. Our data do not show any significant difference between control and patient samples. The haplotypic distribution among patients and controls was equivalent and consequently these microsatellites and SNPs do not seem to affect IgA deficiency susceptibility per se.

A possible caveat that has always to be considered when a negative result is reached is that the sample size may not be large enough and that in consequence we can not see a possible difference between cases and controls. It is our 
Table 3: IL-I OR/IL- I 0G/promoter SNPs haplotypes, transmitted (388) and not transmitted (332)

\begin{tabular}{lcccc}
\hline Haplotype & $\mathrm{T}$ & $\%$ & $\mathrm{NT}$ & $\%$ \\
\hline 2/9/ATA & 66 & 17 & 61 & 18 \\
2/I3/ACC & 55 & 14 & 40 & 12 \\
3/9/GCC & 42 & 11 & 27 & 8 \\
2/II/GCC & 33 & 9 & 37 & 11 \\
2/I3/GCC & 34 & 9 & 15 & 5 \\
2/I4/ACC & 24 & 6 & 27 & 8 \\
2/I2/ACC & 22 & 6 & 12 & 4 \\
3/IO/GCC & 8 & 2 & 11 & 3 \\
3/9/ATA & 15 & 4 & 16 & 5 \\
2/II/ACC & 10 & 3 & 9 & 3 \\
2/I2/GCC & 10 & 3 & 5 & 2 \\
Other & 69 & 18 & 72 & 22 \\
\hline
\end{tabular}

belief that the present study has a reasonable statistical power $(80 \%$ power to detect a relative risk of 1.5 with an allele frequency of 0.1 ) so we can confidently conclude that if there is in fact an effect, it should be a rather small one because our sample is powered enough to detect any clinically relevant effect. Another reason that could explain our negative results is that the polymorphisms we have studied do not have functional relevance, or are not in linkage disequilibrium with relevant polymorphisms located elsewhere in the IL-10 gene. However there are functional studies implicating the position -1082 of the IL-10 promoter and IL-10R/IL-10G microsatellite haplotypes [20] in the production in vitro of IL-10. Moreover, the high degree of linkage disequilibrium and the presence of extended haplotypes [21] make our choice of genetic markers useful to incorporate most of the genetic information present at the IL-10 locus.

As we know, IL-10 has a dual role both as inhibitory and stimulatory component of the immune responses. There are not references in the bibliography reporting that an alteration of IL-10 levels could eventually cause an immunodeficiency. On the contrary, there have been many studies that find genetic associations of the IL-10 polymorphisms with inflammatory diseases like rheumatoid arthritis, type 1 diabetes, systemic lupus erythematosus or inflammatory bowel diseases [19,26-30]. Therefore the evidence suggests that IL-10 deficiency or alteration could be more important as an etiologic factor in autoimmune diseases than in immunodeficiencies. This conclusion is supported by studies with IL-10 knock-out mice, which develop a chronic enterocolitis. The administration of IL10 to these mice ameliorates the symptoms of the disease [15]. Administration of recombinant human IL-10 to colitis patients is being evaluated as a therapeutic option in colitis patients. It may be speculated that the role of IL-10 as stimulatory factor is redundant with that of other cytokines (e.g. IL-6), and therefore a defect in one of its positive effects is not clinically detectable. Only inhibitory functions of IL-10 might be non-redundant.

Therefore we can conclude that, in terms of sample size, genetic markers included, and haplotype analysis, our work greatly expands previous bibliography about the effect of IL-10 polymorphisms on IgAD. It would be really interesting in the future to analyze further the IL-10 locus and its possible interactions with other loci coding for other molecules involved in B cell maturation and final differentiation to plasma cells, like genes coding for IL-10 receptor chains or the gene recently described as associated with IgAD and common variable immunodeficiency, TACI $[31,32]$.

\section{Conclusion}

Our data do not show any significant difference in IL-10 polymorphism frequencies between control and IgAD patient samples. Their haplotype distribution among patients and controls was also equivalent and therefore these microsatellites and SNPs do not seem to influence IgAD susceptibility.

\section{Competing interests}

The author(s) declare that they have no competing interests.

\section{Authors' contributions}

JO performed the genetic and statistical analyses and drafted the manuscript. MFA helped with data acquisition and coordinated the study. EU helped to design the study and revised the manuscript critically. RLM participated in data acquisition and revised critically the manuscript. AF participated in the collection of samples and IgA quantification and participated in the design and coordination of the study. GF participated in the collection of samples and IgA quantification. EGdC participated in the design of the study and revised critically the manuscript. AM conceived the study, participated in its design and coordination, data acquisition and revised critically the manuscript.

\section{Acknowledgements}

We are most grateful to Carmen Martínez Cuervo for her expert technical assistance.

Alfonso Martinez is employee with support from the "Fondo de Investigaciones Sanitarias" (CP04/00 I75) and Elena Urcelay is recipient of a "Ramon y Cajal" contract from the Spanish Science and Technology Ministry. This work was supported by project number PI05I22I from the "Fondo de Investigaciones Sanitarias".

\section{References}

I. Schaffer FM, Monteiro RC, Volanakis JE, Cooper MD: IgA deficiency. Immunodefic Rev I99I, 3(I): I5-44.

2. Wilton AN, Cobain TJ, Dawkins RL: Family studies of IgA deficiency. Immunogenetics 1985, 2 I (4):333-342.

3. Volanakis JE, Zhu ZB, Schaffer FM, Macon KJ, Palermos J, Barger BO, Go R, Campbell RD, Schroeder HW Jr, Cooper MD: Major histo- 
compatibility complex class III genes and susceptibility to immunoglobulin A deficiency and common variable immunodeficiency. J Clin Invest 1992, 89(6): 1914-1922.

4. Cataldo F, Lio D, Marino V, Scola L, Crivello A, Corazza GR: Plasma cytokine profiles in patients with celiac disease and selective IgA deficiency. Pediatr Allergy Immunol 2003, I 4(4):320-324.

5. Klemola T, Savilahti E, Koskimies S, Pelkonen P: HLA antigens in IgA deficient paediatric patients. Tissue Antigens 1988 32(4):218-223.

6. Burrows PD, Cooper MD: IgA deficiency. Adv Immunol 1997, 65:245-276

7. Conley ME, Cooper MD: Genetic basis of abnormal B cell development. Curr Opin Immunol 1998, I 0(4):399-406.

8. Mitsuya H, Tomino S, Hisamitsu S, Kishimoto S: Evidence for the failure of IgA specific $T$ helper activity in a patient with immunodeficiency with hyper IgM. J Clin Lab Immunol 1979, 2(4):337-342.

9. Inoue T, Okubo H, Kudo J, Ikuta T, Hachimine K, Shibata R, Yoshinari $O$, Fukada K, Yanase T: Selective IgA deficiency: analysis of Ig production in vitro. I Clin Immunol I984, 4(3):235-24I.

10. Mocellin S, Panelli MC, Wang E, Nagorsen D, Marincola FM: The dual role of IL-10. Trends Immunol 2003, 24(I):36-43.

1I. Di Carlo E, Coletti A, Modesti A, Giovarelli M, Forni G, Musiani P: Local release of interleukin- $I 0$ by transfected mouse adenocarcinoma cells exhibits pro- and anti-inflammatory activity and results in a delayed tumor rejection. Eur Cytokine Netw 1998, 9(I):61-68.

12. Langenkamp A, Messi M, Lanzavecchia A, Sallusto F: Kinetics of dendritic cell activation: impact on priming of THI, TH2 and nonpolarized T cells. Nat Immunol 2000, I (4):3II-3|6.

13. Moore KW, de Waal Malefyt R, Coffman RL, O'Garra A: Interleukin- 10 and the interleukin- 10 receptor. Annu Rev Immunol 200I, 19:683-765

14. Mantovani A, Sozzani S, Locati M, Allavena P, Sica A: Macrophage polarization: tumor-associated macrophages as a paradigm for polarized M2 mononuclear phagocytes. Trends Immunol 2002, 23(I I):549-555.

15. Kuhn R, Lohler J, Rennick D, Rajewsky K, Muller W: Interleukin- I Odeficient mice develop chronic enterocolitis. Cell 1993, 75(2):263-274.

16. Briere F, Bridon JM, Chevet D, Souillet G, Bienvenu F, Guret C, Martinez-Valdez H, Banchereau J: Interleukin 10 induces B lymphocytes from IgA-deficient patients to secrete IgA. J Clin Invest 1994, 94(1):97-104.

17. Crawley E, Kay R, Sillibourne J, Patel P, Hutchinson I, Woo P: Polymorphic haplotypes of the interleukin- 10 ' flanking region determine variable interleukin- 10 transcription and are associated with particular phenotypes of juvenile rheumatoid arthritis. Arthritis Rheum 1999, 42(6): I I0I-II 08.

18. Eskdale J, Kube D, Tesch H, Gallagher G: Mapping of the human ILI 0 gene and further characterization of the 5' flanking sequence. Immunogenetics 1997, 46(2): I20-128.

19. Eskdale J, Wordsworth P, Bowman S, Field M, Gallagher G: Association between polymorphisms at the human IL-I 0 locus and systemic lupus erythematosus. Tissue Antigens 1997 49(6):635-639.

20. Eskdale J, Gallagher G, Verweij CL, Keijsers V, Westendorp RG, Huizinga TW: Interleukin 10 secretion in relation to human IL- 10 locus haplotypes. Proc Natl Acad Sci U S A 1998, 95( I 6):9465-9470.

21. Eskdale J, Keijsers V, Huizinga T, Gallagher G: Microsatellite alleles and single nucleotide polymorphisms (SNP) combine to form four major haplotype families at the human interleukin-IO (IL-I0) locus. Genes Immun I999, I (2):I5I-I55.

22. Cataldo F, Lio D, Marino V, Scola L, Crivello A, Mule AM, Corazza GR: Cytokine genotyping (TNF and IL-I0) in patients with celiac disease and selective IgA deficiency. Am J Gastroenterol 2003, 98(4):850-856.

23. De la Concha EG, Fernandez-Arquero M, Gual L, Vigil P, Martinez A, Urcelay E, Ferreira A, Garcia-Rodriguez MC, Fontan G: MHC susceptibility genes to IgA deficiency are located in different regions on different HLA haplotypes. J Immunol 2002, I 69(8):4637-4643.

24. Martinez A, Gual L, Fernandez-Arquero M, Nogales A, Ferreira A Garcia-Rodriguez MC, Fontan G, de la Concha Ed EG: Epistatic effects occurring among susceptibility and protective MHC genes in IgA deficiency. Genes Immun 2003, 4(4):316-320.
25. Gual L, Martinez A, Fernandez-Arquero M, Garcia-Rodriguez MC, Ferreira A, Fontan G, de la Concha EG, Urcelay E: Major histocompatibility complex haplotypes in Spanish immunoglobulin A deficiency patients: a comparative fine mapping microsatellite study. Tissue Antigens 2004, 64(6):67I-677.

26. Martinez Doncel A, Rubio A, Arroyo R, de las Heras V, Martin C, Fernandez-Arquero M, de la Concha EG: Interleukin- 10 polymorphisms in Spanish multiple sclerosis patients. J Neuroimmunol 2002, I 3 I (I-2): I68-I72.

27. Martinez A, Pascual M, Pascual-Salcedo D, Balsa A, Martin J, de la Concha EG: Genetic polymorphisms in Spanish rheumatoid arthritis patients: an association and linkage study. Genes Immun 2003, 4(2): I|7-I2I.

28. Cavet J, Middleton PG, Segall M, Noreen H, Davies SM, Dickinson AM: Recipient tumor necrosis factor-alpha and interleukin10 gene polymorphisms associate with early mortality and acute graft-versus-host disease severity in HLA-matched sibling bone marrow transplants. Blood I999, 94(I I):394I-3946.

29. Urcelay E, Santiago JL, de la Calle H, Martinez A, Figueredo A, Fernandez-Arquero $M$, de la Concha EG: Interleukin-10 polymorphisms in Spanish type I diabetes patients. Genes Immun 2004, 5(4):306-309.

30. Fernandez L, Martinez A, Mendoza JL Urcelay E, Fernandez-Arquero M, Garcia-Paredes J, Diaz-Rubio M, de la Concha EG: Interleukin- 10 polymorphisms in Spanish patients with IBD. Inflamm Bowel Dis 2005, I I (8):739-743.

31. Salzer U, Chapel HM, Webster AD, Pan-Hammarstrom Q, SchmittGraeff A, Schlesier M, Peter HH, Rockstroh JK, Schneider P, Schaffer AA, Hammarstrom L, Grimbacher B: Mutations in TNFRSFI3B encoding TACl are associated with common variable immunodeficiency in humans. Nat Genet 2005, 37(8):820-828.

32. Castigli E, Wilson SA, Garibyan L, Rachid R, Bonilla F, Schneider L, Geha RS: TACI is mutant in common variable immunodeficiency and IgA deficiency. Nat Genet 2005, 37(8):829-834.

\section{Pre-publication history}

The pre-publication history for this paper can be accessed here:

http://www.biomedcentral.com/1471-2350/7/56/prepub
Publish with Bio Med Central and every scientist can read your work free of charge

"BioMed Central will be the most significant development for disseminating the results of biomedical research in our lifetime. "

Sir Paul Nurse, Cancer Research UK

Your research papers will be:

- available free of charge to the entire biomedical community

- peer reviewed and published immediately upon acceptance

- cited in PubMed and archived on PubMed Central

- yours - you keep the copyright

Submit your manuscript here:

http://www.biomedcentral.com/info/publishing_adv.asp
BioMedcentral 